\title{
L'argomentazione politica in Antirrēsis di Hipacy Pociej
}

Abstract: Nosilia Viviana, L'argomentazione politica in Antirrēsis di Hipacy Pociej (The Political Argumentation in Hipacy Pociej's Antirrēsis). "Poznańskie Studia Slawistyczne" 10. Poznań 2016. Publishing House of the Poznań Society for the Advancement of the Arts and Sciences, pp. 213-225. ISSN 2084-3011.

The article investigates the interconfessional polemical literature as a valuable source offering an insight into the major values of the society of the Polish-Lithuanian Commonwealth in the $16^{\text {th }}-17^{\text {th }}$ centuries. These texts also help understanding phenomena other than the merely religious ones. In the debate, juridical and political arguments were used too. We focus on the case study of Hipacy Pociej's Antirrēsis (Ruthenian: 1599; Polish: 1600), written in response to Marcin Broniewski's Apokrisis (1597). Pociej, who had an outstanding political career before becoming a Uniate bishop, mastered these arguments perfectly. His work is imbued with the mentality of the communitas nobilium, which also played a part in his lexical choices.

KeYwords: Hipacy Pociej; Orthodox-Uniate polemics; Uniate Church in the $16^{\text {th }}-17^{\text {th }}$ centuries; art of argumentation; Polish-Lithuanian Commonwealth; interconfessional relationships

Nel repertorio argomentativo utilizzato dai polemisti nella discussione legata all'Unione di Brest ${ }^{1}$ trovavano ampio spazio le considerazioni di carattere giuridico ed anche politico. Le argomentazioni giuridiche sono particolarmente frequenti nella fase iniziale, quando ciascuna delle due ormai distinte Chiese di rito greco-bizantino nella Respublica ${ }^{2}$ cercava di

${ }^{1}$ Città oggi in Bielorussia, in polacco: Brześć. Utilizziamo qui il nome nella versione bielorussa, coincidente con quella russa, perché è quella che si è imposta nella formula con cui nella letteratura scientifica occidentale si indica la serie di eventi che hanno portato, fra il 1595 e il 1596, al riconoscimento della giurisdizione di Roma da parte di rappresentanti della gerarchia rutena.

${ }^{2}$ Per indicare lo Stato polacco-lituano useremo talora l'espressione Respublica, talaltra l'espressione della lingua originale, Rzeczpospolita, per pura variatio, per segnalare la particolarità del regime politico di questo Paese, che era una monarchia con un parlamento dagli ampi poteri. 
dimostrare la propria legittimità e la titolarità dei diritti di godimento sui beni e di prerogative concessi in passato ai cittadini di „fede greca" ${ }^{3}$. Le questioni giuridiche e politiche tornano in primo piano quando gli ortodossi $^{4}$ cercano di legittimare la nuova gerarchia consacrata dal patriarca di Gerusalemme Teofane (1620-1621) e in occasione delle contrattazioni precedenti l'incoronazione di Władysław IV ${ }^{5}$ (cf. Bortnik 2007: 163-165).

Per quanto riguarda la valenza politica delle opere polemiche, già la loro stessa pubblicazione e la scelta del dedicatario erano dichiarazioni politicamente rilevanti di per sé. Non mancano però anche riferimenti più diretti, che miravano ad indirizzare le scelte dei potenti. Inoltre, il modo di proporre l'argomentazione e di esprimersi lasciavano trapelare anch'essi la visione ideologica dello scrittore.

Un caso interessante è quello di Hipacy (al secolo Adam) Pociej ${ }^{6}$. Il cursus honorum di Pociej lo aveva portato ad occupare lo scranno di senatore. Anche dopo avere rinunciato a quella posizione per contribuire al risanamento della Chiesa di rito bizantino-slavo, divenendo vescovo di Volodymyr Volyns' $\mathrm{kyj}^{7}$ (in Volinia), egli non riuscì a deporre completamente i suoi costumi retorici e linguistici, né il suo abito mentale.

${ }^{3}$ Ovvero ai fedeli di rito orientale.

${ }^{4}$ La terminologia per definire i fedeli delle diverse confessioni non coincideva con quella attuale. Userò tuttavia quest'ultima per semplicità.

${ }^{5} \mathrm{Cf}$. l'eccellente panoramica della letteratura polemica scritta da Jan Stradomski (2003: 30-50).

${ }^{6}$ Ucraino: Іпатій Потій; bielorusso: Іпацій Пацей. Per i nomi di persona userò nel testo la versione polacca, in quanto proprio l'appartenenza alla Rzeczpospolita polacco-lituana costituiva l'elemento che accomunava personaggi la cui storia è legata a regioni molto diverse. Adam Pociej nacque nel 1541 da una famiglia nobile; si formò in una scuola calvinista. La sua carriera politica lo portò a divenire castellano e senatore (1588). Nel 1574 abbandonò il Calvinismo, che aveva abbracciato durante la gioventù, e tornò all'Ortodossia. Rimasto vedovo, per iniziativa del principe Konstanty Ostrogski divenne monaco col nome di Hipacy e, nel 1593, vescovo di Volodymyr e Brest. Re Zygmunt III raccomandò caldamente Adam Pociej al metropolita Rahoza, sottolineando i suoi nobili natali, la sua rispettabilità e la sua abilità di scrittore in una lettera del 20 marzo 1593 (AZR 4, n. 44, p. 63). Nel 1595 si recò a Roma per sancire l'unione della Chiesa rutena con la Chiesa di Roma. Alla morte del metropolita di Kyjiv Rahoza, divenne metropolita della Chiesa rutena unita a Roma (1600). Morì nel 1613. Cf. Lencyk, Nazarko 1993; Byliński, Długosz 1997: 6-7; Stradomski 2003: 36; Dzięgielewski 1983: 28-34.

${ }^{7}$ In polacco: Włodzimierz Wołyński; oggi città ucraina. Qui e di seguito userò per i nomi geografici di cui non esiste o non è diffuso un corrispettivo italiano la denominazione odierna. 


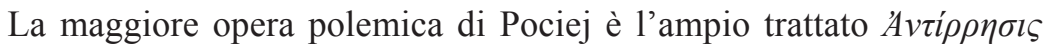
abo Apologija przeciwko Krzysztofowi Philaletowi..., pubblicato a Vilna dapprima in ruteno (1599) e poi anche in polacco (1600) (Byliński, Długosz 1997: 28). Quest'opera, pubblicata anonima, costituiva una diretta risposta al libro Apokrisis..., scritto su incarico del principe Konstanty Ostrogski ${ }^{8}$ dal protestante Marcin Broniewski, che era stato in precedenza assoldato dal magnate anche per comporre un resoconto dal punto di vista degli ortodossi degli eventi dei due sinodi tenutisi a Brest nel 1596, resoconto pubblicato in polacco a Cracovia nel 1597 e riedito nello stesso anno a Vilna in polacco e ruteno (Byliński, Długosz 1997: 26-29; Stradomski 2003: 32-34). Broniewski aveva pubblicato Apokrisis sotto uno pseudonimo, Christophor Philalet, per non rivelare la sua appartenenza confessionale, che tuttavia trapela molto chiaramente dai suoi scritti, offrendo così il destro al suo oppositore per screditarlo agli occhi dei lettori (Stradomski 2003: 32-33; cf. Pociej 1997: 46).

Pociej dedica la sua opera polemica capitale al gran cancelliere di Lituania Lew (Leon) Sapieha ${ }^{9}$, che era stato presente al Sinodo unionista di Brest in qualità di messo del re (Byliński, Długosz 1997: 23), ma che un quarto di secolo dopo avrebbe manifestato le sue perplessità sull'Unione e sulle sue conseguenze per lo Stato polacco-lituano (Byliński, Długosz 1997: 36). Nella dedica Pociej non si diffonde a lungo nell'elogio del dedicatario preferendo invece ingaggiare subito la polemica cominciando da un'apparentemente oziosa disputa... sulle dediche! Pociej accusa Christophor Philalet di avere sfrontatamente dedicato al gran cancelliere della Corona Jan Zamoyski, cattolico, il suo libello contenente concezioni „eretiche" (ovvero protestanti, secondo la terminologia dell'epoca). Il tentativo di Philalet di associare la propria opera al nome di una delle cariche più importanti dello Stato, appartenente ad una confessione rivale, equivaleva nella percezione di Pociej ad una intollerabile offesa. Nell'Antirrēsis il prelato uniate pubblica una lettera di Jan Zamoyski diretta a lui nella quale il cancelliere afferma di non avere mai sentito parlare del libro con la de-

${ }^{8}$ Ucraino: Костянтин Острозький. Su di lui si veda la monografia di Tomasz Kempa: Konstanty Wasyl Ostrogski (ok. 1524/1525-1608). Wojewoda kijowski i marszałek Ziemi Wotyńskiej (1997).

${ }^{9}$ Lituano: Leonas Sapiega; bielorusso: Леў Сапега. 
dica ,incriminata" e di voler prendere provvedimenti contro il suo autore (Pociej 1997: 43).

Già solo con la questione delle dediche Pociej persegue più obiettivi:

1) getta discredito sull'avversario, mettendo in rilievo la sua irrispettosità verso le alte cariche dello Stato, il che equivaleva a far considerare Broniewski un individuo sospetto;

2) riportando la lettera del gran cancelliere Jan Zamoyski al vescovo di Volodymyr (ovvero a lui stesso), Pociej ostenta la sua familiarità con un personaggio di tale rilievo e suggerisce la benevolenza verso la gerarchia ecclesiastica uniate da parte delle massime cariche del regno, facendo apparire la Chiesa uniate come dominio dell'ordine e in armonia con le istituzioni.

In Antirrēsis viene chiamata in causa anche un'altra figura importantissima per il panorama sia politico sia religioso del periodo: il principe Konstanty Ostrogski, voivoda di Kyjiv. Broniewski aveva citato brani della corrispondenza fra i prelati ruteni ed il principe; Pociej decide di pubblicare in extenso le lettere rilevanti del suo scambio epistolare col magnate, per far sì che il lettore possa confrontarsi con documenti interi, non con brani estrapolati dal contesto.

La descrizione del carteggio e delle circostanze che lo avevano accompagnato doveva smentire l'accusa di Broniewski ai prelati uniati di avere agito volutamente di nascosto, addirittura ingannando il principe sulle loro reali intenzioni. In effetti, le lettere del metropolita Rahoza ${ }^{10}$ riportate da Broniewski sono sotto certi aspetti evasive: il metropolita assicura il principe di non voler introdurre nulla di nuovo nella Chiesa orientale, ma ciò non esclude affatto che egli fosse impegnato in trattative per addivenire all'Unione, perché, secondo il pensiero degli uniati, con l'Unione si preservava l'antico. Anche le dichiarazioni sull'immutato rispetto verso il patriarca di Costantinopoli non potevano dirsi del tutto false, perché il cambiamento di giurisdizione non significava negare ogni riconoscimento onorifico alle altre antiche sedi patriarcali. Nella terza lettera ad Ostrogski (28 IX/8 X 1595), Rahoza nega di avere già posto i fedeli di rito orientale sotto la giurisdizione di Roma (Broniewski 1882: 1059-1060): in effetti,

${ }^{10}$ Bielorusso: Міхаіл Рагоза, primo metropolita uniate di Kyjiv (1596-1600), fautore dell'Unione di Brest. Cf. la circolare di re Zygmunt III, AZR 4, n. 19, pp. 25-26. 
egli si apprestava a partire per Roma e non aveva ancora prestato formalmente atto di obbedienza. I brani delle lettere del metropolita riportati da Broniewski creano davvero l'impressione che il prelato avesse dato prova di astuzia gesuitica.

Pociej si trova a dover dimostrare la buona fede degli uniati e in particolare la sua, dato che era stato accusato di avere partecipato alla cospirazione. Egli non si limita a discolparsi, ma divulga anche il messaggio che il principe aveva fatto pervenire ai protestanti, in cui egli si dichiarava pronto ad intervenire anche con il suo esercito privato per contrastare i progetti di Unione. Il documento era già stato intercettato e portato a conoscenza del re, generando manifestazioni di biasimo che Pociej non manca di inserire nel suo scritto (Pociej 1997: istruzione rivolta al sinodo evangelico di Toruń ${ }^{11}$ : 104-108; lettera di biasimo di un senatore: 108-110). Pubblicando i documenti, Pociej cerca di compromettere il principe di fronte agli ortodossi, per privarli del loro più potente patrono laico. Ostrogski, inoltre, viene presentato come turbator pacis del regno.

Nel contesto della polemica con Broniewski, l'argomento della concordia all'interno della Rzeczpospolita era particolarmente rilevante, poiché l'autore di Apokrisis vi aveva fatto ricorso ampiamente e con particolare incisività. La serrata argomentazione di Broniewski culmina nella sua conclusiva „supplica e ammonizione” ai cittadini (obywatele) della Corona e del Gran Principato di Lituania. Già la scelta del termine obywatele (e non, p. es., wierni - fedeli) determina il tono del discorso, che è intessuto completamente a partire da argomenti di carattere giuridico e politico. Broniewski rileva come le discordie a sfondo religioso siano degenerate in altri Stati in guerre intestine (Broniewski 1882: 1797). Le conseguenze di una tanto temuta guerra civile (wojna wewnętrzna) in Polonia sarebbero molto più esiziali, a causa della prossimità con pericolosi aggressori (1801-1802). La compressione dei diritti garantiti in precedenza ad una parte significativa della popolazione, con la palese violazione di ogni giuramento, legge ed accordo, provoca la disaffezione nei confronti dello Stato (1812). Broniewski sottolinea che la tutela della Chiesa

\footnotetext{
${ }^{11}$ Sui tentativi di abboccamento fra ortodossi e protestanti si vedano i lavori di Tomasz Kempa, in particolare la monografia Wobec kontrreformacji. Protestanci i prawosławni $w$ obronie swobód wyznaniowych $w$ Rzeczpospolitej $w$ końcu XVI $i$ w pierwszej połowie XVII wieku (2007).
} 
ortodossa non era solo una questione privata, bensì un problema di sicurezza nazionale (1814). L'autore afferma di avere scritto la sua opera ,per benevolenza e amore verso la patria comune" (1815), dopo avere ricordato che la fedeltà alla Chiesa ortodossa e ai patriarchi orientali non aveva mai impedito ai ruteni in passato di essere leali verso la Rzeczpospolita (1801). Gli ortodossi erano disposti a sacrificare al re il loro corpo ed i loro averi, ma la loro anima era votata soltanto al Re dei Cieli (1808; cfr. anche Naumow 2002: 69).

Pociej era obbligato a servirsi dello stesso tipo di argomentazione e a rivolgersi allo stesso pubblico che aveva chiamato in causa il suo opponente. Se il ricorso ad un'argomentazione di carattere politico e giuridico è dettato dall'avversario polemico, Pociej dimostra però di padroneggiare perfettamente gli strumenti per condurre una disputa di questo tenore. Lo scontro fra Broniewski e Pociej si segnala per l'approccio legale-giuridico ai fatti.

Broniewski aveva accusato la gerarchia ecclesiastica uniate di avere violato il giuramento prestato al patriarca di Costantinopoli al momento della consacrazione e sostiene che per tale ragione i vescovi erano stati deposti dal Sinodo ortodosso (Broniewski 1882: 1037-1040). L'accusa di spergiuro era grave, perché si trattava di una seria violazione dell'etica sociale. Pociej replica con argomenti capziosi da corte tribunalizia: egli osserva che all'epoca dei due sinodi di Brest del 1596 la sede patriarcale era da considerare vacante e che, come avveniva nei periodi di interregno nella Rzeczpospolita, non era possibile deporre nessuno dal suo incarico (Pociej 1997: 54-57). Entrambi i contendenti applicano gli stessi schemi dell'amministrazione secolare alle procedure ecclesiastiche. Essi attribuiscono molta importanza alle questioni procedurali, formali, come la trascrizione degli atti nei registri ufficiali (p. es. Broniewski 1882: 1025-1026) e l'apposizione di sigilli e firme sui documenti (Pociej 1997: 64).

Entrambi cercano di provare la legittimità dell'operato e dei diritti delle Chiese di rito orientale ormai separate, adducendo provvedimenti legislativi che ciascuno dei polemisti interpretava in favore della parte rappresentata.

Oggetto di particolare discussione era la Confederazione di Varsavia (23 I 1573), che aveva garantito la libertà di culto nelle terre facenti parte dei possedimenti del re. Il provvedimento era stato varato per dirimere 
i conflitti fra i protestanti e i cattolici, ma Broniewski cerca di mostrarne l'applicabilità anche agli ortodossi (Broniewski 1882: 1075-1076; Chodynicki 2005: 357-358). Pociej, che pure era stato calvinista in gioventù, manifesta la sua disapprovazione per questa legge, che aveva, ,aperto a tutte le eresie le porte per il male" (Pociej 1997: 173). Proprio l'adozione di questa legge sulla tolleranza religiosa avrebbe permesso il diffondersi di „eresie" e dell'arbitrio (Pociej 1997: 111), che era visto come una gravissima minaccia nel dibattito politico dell'epoca. Esercitare l'arbitrio (swawola) col pretesto di godere della libertà (wolność), fondamento dello Stato nobiliare, era un comportamento generalmente riprovevole per il suo elevato potenziale destabilizzante ${ }^{12}$.

Pociej doveva difendere gli uniati dall'accusa di avere violato la aurea libertas. Si trovava in una situazione particolarmente difficile. La Chiesa uniate era quella sostenuta (seppur con minore zelo di quanto avrebbe voluto Pociej) dal re e da molti notabili del regno ${ }^{13}$ ed era considerata erede dei diritti concessi in passato ai ruteni in generale. In qualunque altra monarchia europea coeva sarebbe stato naturale attendersi un sostegno concreto molto deciso da parte delle autorità secolari per imporre l'applicazione delle scelte del re, ma la Polonia era un contesto del tutto particolare. Pociej sapeva molto bene che un argomento del genere sarebbe stato controproducente, data l'avversione del ceto nobiliare della Rzeczpospolita verso ogni possibile minaccia alla sua venerata libertà. Egli sceglie così una strategia argomentativa diversa: accusa gli ortodossi che ora si schieravano così attivamente contro la gerarchia ecclesiastica uniate di essere stati politicamente inerti e di non essersi adoperati nelle sedi di loro competenza per esigere l'applicazione dei provvedimenti in favore della ,fede greca”. In passato la gerarchia ecclesiastica ortodossa era stata negligente anche perché aveva trascurato di portare avanti l'opera benemerita avviata dal Concilio di Firenze e che aveva condotto all'Unione di Chiesa latina e greca. Conseguenza diretta dell'Unione di Firenze era stata l'emanazione del privilegio di re Władysław Jagiellończyk del 1443 (Chodynicki 2005: 95),

${ }^{12} \mathrm{Nel}$ dibattito religioso della Reczpospolita la contrapposizione tra swawola e wolność resterà anche in seguito una componente stabile del repertorio argomentativo.

${ }^{13} \mathrm{Si}$ veda la prefazione al lettore nel trattato di Pociej O przywilejach nadanych od Najjaśnieyszych Królów Polskich..., Wilno 1606? (traggo luogo e possibile data di edizione da Stradomski 2003: 297). 
in cui la gerarchia ecclesiastica di rito greco che si era unita alla Chiesa di Roma era equiparata a quella di rito latino (Pociej 1997: 213-214). La negligenza (niedbatość) sul piano dell'Unione religiosa si era ripercossa sul piano dello status giuridico della Chiesa rutena, che, sottrattasi alla giurisdizione di Roma, aveva visto cadere i presupposti per l'attuazione del provvedimento, fino a quando non aveva ricostituito la condizione di unità negli anni 1595-1596 (215). Anche nella lettera al principe Ostrogski del 3 VI 1598 pubblicata in Antirrēsis Pociej sottolinea il collegamento tra l'Unione ecclesiale ed il godimento dei diritti (223). La restaurazione dell'Unione avrebbe condotto al ripristino dei diritti - non solo religiosi dei ruteni.

Pociej, come tutti i polemisti favorevoli all'Unione di Brest, sottolinea come essa ricostituisse una situazione precedente e non introducesse nulla di nuovo ${ }^{14}$. La diffidenza verso l'introduzione delle novità era prevedibile nel dibattito riguardante la Chiesa, che si poneva come attenta custode della tradizione formatasi nel passato e all'epoca di Pociej era particolarmente timorosa delle novità dopo il trauma causato dalla Riforma. Nel discorso pubblico della Rzeczpospolita, però, queste rassicurazioni erano ancora più importanti per la peculiarità della struttura istituzionale dello Stato polacco-lituano, in cui la centralità del parlamento, in particolare dall'approvazione della norma riassunta nella formula nihil novi sine communi consensu (1505), richiedeva un consenso molto ampio per poter varare provvedimenti. Per una classe dirigente abituata a questo tipo di funzionamento delle istituzioni, doveva essere naturale attendersi che un principio analogo fosse applicato anche negli altri aspetti della vita pubblica ${ }^{15}$. Del resto, anche nella Chiesa orientale tale principio era importantissimo nella pratica ecclesiastica. In tale contesto, 1'Unione di Brest, che non era fondata su un ampio consenso, doveva apparire sospetta. Per i polemisti che promuovevano la causa dell'Unione sostenere la tesi della sua continuità con il passato era vantaggioso sotto tutti i punti di vista.

Pociej si trovava però in grave imbarazzo: l'equiparazione dei diritti della Chiesa uniate a quella latina non era avvenuta, e i presuli uniati non

\footnotetext{
${ }^{14}$ Sul modo di concepire l'unità della Chiesa e sulla sua importanza per Pociej cf. Ozorowski 2012: 152-164.

${ }^{15}$ Sulle tendenze fortemente conservatrici nelle istituzioni polacche del XVII secolo $\mathrm{cf}$. Olszewski 1977, in particolare le pp. 60-61, 67-70, 88-89.
} 
avevano ottenuto un posto in senato; Broniewski non aveva mancato di sottolineare questa circostanza, come pure l'anonimo polemista ortodosso che si firmava „Chierico di Ostroh”". L'autore di Antirrēsis si trae d'impaccio ancora spostando la discussione su un orizzonte valoriale profano. Pociej cerca di convincere della certa realizzazione di quanto promesso presentando come garanzia la parola del re. Dubitare delle promesse del re equivaleva a ledere il suo onore (Pociej 1997: 200; 266-267).

Nello scontro polemico tra Philalet-Broniewski e Pociej la figura del re è menzionata molto spesso. Sono riportati privilegi e lettere di sovrani del passato e di quello attuale. Per la popolazione di rito orientale la vita della Chiesa sotto il dominio di un re di fede diversa rappresentava una situazione difficilmente accettabile (Naumow 2002: 64). Nella Rzeczpospolita si elaborò una concezione che di fatto rappresentava un compromesso, sottolineando la distinzione tra investitura sacrale e diritto di conferire i beni al nuovo metropolita e ai vescovi. Il re era visto come un patrono laico per eccellenza. Veniva enfatizzato il suo ruolo di garante della aurea libertas, una libertà che si estendeva anche, secondo protestanti ed ortodossi, sul piano religioso (Naumow 2002: 68-69). In Polonia il quadro era complicato ulteriormente dal fatto che i re erano elettivi e dovevano di volta in volta accettare le condizioni poste dalla szlachta (la nobiltà di sangue) per poter salire al trono, come rimarca Broniewski (Naumow 2002: 69; Broniewski 1882: 1810).

Pociej, ex senatore del regno, si dimostra particolarmente sensibile al ruolo di garanzia del re e alla struttura gerarchica della società. La presenza dei messi del re al Sinodo unionista di Brest è usata da lui come prova della sua legittimità (Pociej 1997: 114 e passim). Pociej accusa Broniewski in più occasioni di mancare di rispetto all'autorità suprema del regno (Pociej 1997: 114, 205, 208-210, 212). Il re, benché di confessione diversa, è comunque investito di una certa sacralità: egli è il ,signore pio e santo” (114) e „l'unto del Signore” (pomazaniec Boży, cf. p. es. p. 50, 201, 206, 208), espressione che sarà condivisa anche dai polemisti ortodossi (Naumow 2002: 69-70).

\footnotetext{
${ }^{16}$ Polemista ortodosso anonimo, autore, fra l'altro, di un'opera polemica in ruteno sul Concilio di Firenze, Исторія о листрикійскомъ... синодъ (Ostroh 1598) (Stradomski 2003: 170). L'opera è ripubblicata in Русская историческая библиотека, XIX, Петербургъ 1903, coll. 433-476.
} 
La particolare dignità del re discendeva anche su tutte le istituzioni pubbliche della Rzeczpospolita (come la cancelleria regia; cf. Pociej 1997: 201).

Queste constatazioni conducono ad un altro punto controverso: il ruolo dei laici nel processo decisionale della Chiesa. Anche in questo caso gli uniati sono in difficoltà: nella tradizione della Chiesa orientale ai concili la partecipazione dei laici e del clero di rango inferiore era molto ampia. Nella polemistica ortodossa questa tradizione era continuamente ricordata: Broniewski in Apokrisis coniuga l'argomentazione ispirata alla tradizione ortodossa con le sue idee protestanti sul ruolo attivo dei laici nell'interpretazione delle Scritture. Per gli uniati si pone un'altra difficile sfida: come conciliare la fedeltà alla tradizione orientale con la composizione non canonica del Sinodo di Brest (cf. Stradomski 2003: 30)? Di nuovo Pociej è costretto all'equilibrismo retorico. La strategia che sceglie è quella di rivedere l'interpretazione della tradizione, cercando di sminuire il ruolo degli spesso menzionati imperatori nei concili antichi e di sottolineare l'alto rango dei pochi laici presenti al Sinodo uniate di Brest (Pociej 1997: 62-70; 154-156).

L'attenzione viene da lui spostata su questioni controverse nella composizione del Sinodo rivale, ovvero sul clamoroso caso del protosynkellos ( $\pi \rho \omega \tau о \sigma u ́ \gamma \kappa \varepsilon \lambda \lambda \circ \varsigma)$ Niceforo e dei rappresentanti greci (Pociej 1997: 70). Gli ortodossi sono accusati di avere introdotto nella Rzeczpospolita personaggi sospetti e di continuare a proteggerli, malgrado essi siano sottoposti a giudizio dalle istituzioni del regno (cf. Stradomski 2003: 219).

Pociej respinge e attribuisce agli avversari l'accusa di essere turbatores pacis che è argomento tradizionale della polemica di parte antiuniate (Pociej 1997: 211). L'Unione è presentata come fattore di ricostituzione dell'ordine, di ricompattamento della società. Pociej è ben consapevole dell'impatto dell'argomento dell'unità e della pace interna della Rzeczpospolita nel discorso pubblico della sua epoca (Pociej 1997: 210-219).

Oltre alle scelte argomentative, in Antirrēsis colpisce anche la veste linguistica, che di nuovo tradisce la consuetudine di Pociej con il mondo delle istituzioni laiche. Egli utilizza numerose espressioni latine che appartengono al linguaggio giuridico-cancelleresco ${ }^{17}$ : extra forum, absque

${ }^{17}$ Anche nel raffigurare il giudizio individuale dell'anima dopo la morte Pociej nelle sue omelie ricorre ad una rappresentazione che rispecchia i processi terreni (Ozorowski 2012: 232). 
legitimo iudice (Pociej 1997: 72), competentes iudices (73), ea lege (156), distinctim (159), in senatu (200)...

Pociej lascia trapelare nei suoi scritti la sua forma mentis tipica dell'uomo di nobili natali (szlachetnie urodzony) che è cosciente e fiero del suo stato e condivide pienamente il modello di società della Respublica nobiliare. Ciò risulta particolarmente evidente nella risposta al Chierico di Ostroh inserita a conclusione di Antirrēsis: l'autore sottolinea di non avere aderito all'Unione con Roma solo per ottenere il seggio in senato, perché egli in passato aveva già goduto di questo privilegio, in virtù dei suoi nobili natali, e vi aveva rinunciato per devozione alla causa. Egli apostrofa brutalmente il suo anonimo avversario, affermando che questi non è degno di rimbrottare un nobile, poiché è di oscuri natali (Pociej 1997: 267).

Nel suo trattato Pociej mostra una particolare suscettibilità nei confronti di ogni offesa alla cnota e alla uczciwość, valori fondanti della società nobiliare, solo parzialmente traducibili con 'virtù' e 'onestà'. Entrambi questi termini sono etimologicamente e concettualmente legati alla parola cześć ('onore'). La cnota poteva avere diverse accezioni, ma, come scrive R. Pawelec, „la cnota su cui si insiste in gran parte dei testi polacchi del XVI e XVII secolo è essenzialmente legata all'interiorizzazione delle norme morali e consuetudinarie" (2011: 174). Secondo le concezioni etico-sociali dell'epoca, la parola si riferiva quasi esclusivamente ai nobili (Pawelec 2011: 169). Anche la parola uczciwość (honestas) è utilizzata da Pociej in quest'accezione connotata da un punto di vista sociale, così come gli altri aggettivi e sostantivi legati per etimologia e significato a queste parole: cny, zacny, cnotliwy, uczciwy ('virtuoso', 'onesto'); zacność ('virtuosità'), poczciwość ('onestà') ${ }^{18}$. Gli aggettivi cny e zacny, fuori dal contesto della Rzeczpospolita, sono usati da Pociej per riferirsi a soggetti di cui valuta positivamente le caratteristiche morali, come il patriarca di Costantinopoli Geremia Tranos (Pociej 1997: 57) ${ }^{19}$.

\footnotetext{
${ }^{18}$ Per una trattazione più compiuta delle sfumature semantiche di questi termini rimando a Pawelec 2011.

${ }^{19}$ Questi termini, con adattamento morfologico e grafico, sono presenti anche nella variante rutena del testo. Non solo, essi ricorrono anche in testi di altri polemisti che scrivevano nella cosiddetta prosta mova. Questa lingua aveva dunque assorbito ed assimilato il lessico dei valori fondamentali della società della Repubblica nobiliare, in cui la szlachta rutena era pienamente inserita.
} 


\section{$* * *$}

La letteratura polemica interconfessionale, pur con tutti i vincoli connaturati a questo particolare tipo di produzione scritta, si rivela un materiale interessantissimo anche per studi che esulano dall'ambito strettamente religioso. Lo scontro polemico costringeva in un certo senso a confrontarsi sui valori essenziali, perché era facendo appello ad essi che si poteva sperare di guadagnare il consenso del complesso pubblico cui ci si rivolgeva, benché fosse concesso ampio spazio anche alle esibizioni di erudizione e di maestria nell'arte retorica. Da questi testi si possono evincere, con la dovuta cautela, informazioni sull'orizzonte assiologico dei singoli partecipanti al dibattito e della società in cui vivevano. Inoltre, queste opere sono anche testimonianze rilevanti dal punto di vista stilistico e linguistico della polemistica barocca.

Il caso di Antirrēsis di Hipacy Pociej è molto significativo. Il polemista ruteno padroneggia perfettamente le regole della disputa letteraria. Nel suo modo di argomentare riconosciamo distintamente la visione politica e sociale del ceto nobiliare, visione che fungeva da elemento unificante nella multietnica Respublica delle Due Nazioni (Naumow 2002: 12) ed era un fattore di inclusione sociale, indipendentemente dalle questioni etniche e religiose. Questa forma mentis è particolarmente evidente nel caso di Pociej, che, grazie alla sua carriera secolare, possedeva un senso dell'onore e una coscienza della dignità del ceto nobiliare particolarmente sviluppati e conosceva molto bene i meccanismi e le consuetudini che regolavano la vita istituzionale della Rzeczpospolita.

\section{Letteratura}

\section{Abbreviazioni}

AZR 4 = Акты, относящиеся к истории Западной России..., т. IV, Санкт-Петербург 1851.

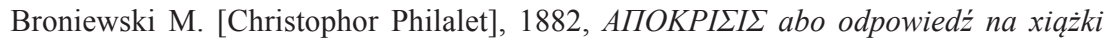
o synodzie brzeskim..., in: Русская историческая библиотека, т. VII, Петербургъ, coll. 1003-1820.

Bortnik J., 2007, Problem tolerancji w prawosławnej myśli teologicznej i spoleczno-politycznej w Rzeczpospolitej drugiej połowy XVI i pierwszej połowy XVII wieku, „Odrodzenie i Reformacje w Polsce” nr 51, pp. 151-175. 
Byliński J., Długosz J., 1997, Wstęp, in: H. Pociej, Antirresis abo Apologija przeciwko Krzysztofowi Philaletowi..., Wrocław, pp. 5-38.

Chodynicki K., 2005, Kościót prawosławny a Rzeczpospolita Polska 1370-1632, Warszawa (prima ed. 1934).

Dzięgielewski J., 1983, Pociej (Potij) Adam, później Hipacy, in: Polski słownik biograficzny, XXVII, Wrocław ecc., pp. 28-34.

Kempa T., 1997, Konstanty Wasyl Ostrowski (ok. 1524/1525-1608). Wojewoda kijowski i marszałek Ziemi Wotyńskiej, Toruń.

Kempa T., 2007, Wobec kontrreformacji. Prostestanci i prawosławni w obronie swobód wyznaniowych w Rzeczpospolitej w końcu XVI $i$ w pierwszej połowie XVII wieku, Toruń.

Lencyk W., Nazarko I., 1993, Potii, Ipatii, in: Encyclopedia of Ukraine, vol. 4, Toronto, $<$ http://www.encyclopediaofukraine.com/display.asp?linkpath=pages $\% 5 \mathrm{CP} \% 5 \mathrm{C}$ O\%5CPotiiIpatii.htm>, 19.02.2016.

Naumow A., 2002, Domus divisa, Studia nad literatura ruska w I. Rzeczypospolitej, Kraków.

Olszewski H., 1977, Ustrój polityczny Rzeczypospolitej, in: Polska XVII wieku, Państwo - społeczeństwo - kultura, a cura di J. Tazbir, Warszawa, pp. 56-89.

Ozorowski M., 2012, Hipacego Pocieja podstawy unickiej teologii pozytywno-polemicznej, Warszawa.

Pawelec R., 2011, Żywoty ludzi poczciwych, skarbu cnoty strzegących. Cześć i cnota, in: Humanizm w języku polskim, Wartości humanistyczne w polskiej leksyce i refleksji o języku, a cura di A. Janowska, M. Pastuchowa, R. Pawelec, Warszawa, pp. 149-174.

Pociej H., 1997, Antirresis abo Apologija przeciwko Krzysztofowi Philaletowi, który niedawno wydat książki imieniem starożytnej Rusi religiej greckiej przeciw książkom o synodzie greckim, napisanym w roku pańskim 1597, oprac. J. Byliński, J. Długosz, Wrocław (prima ed. in ruteno: Вильна 1599; prima ed. in polacco: Wilno 1600).

Stradomski J., 2003, Spory o „,wiarę grecka” w dawnej Rzeczypospolitej, Kraków. 